\title{
About solutions of nonlinear algebraic system with two variables
}

\author{
Rakhshanda Dzhabarzadeh \\ İnstitute Mathematics and Mechanics of NAN of Azerbaijan, Baku
}

Email address:

rakhshanda.dzhabarzade@rambler.ru (R. Dzhabarzadeh)

To cite this article:

Rakhshanda Dzhabarzadeh. About Solutions of Nonlinear Algebraic System with Two Variables. Pure and Applied Mathematics Journal. Vol. 2, No. 1, 2013, pp. 32-37. doi: 10.11648/j.pamj.20130201.15

\begin{abstract}
For nonlinear algebraic system with two variables sufficient conditions of existence of solutions are given. The proof of these statements is received as a corollary of more common reviewing considered in this paper. In particular, in this work the existence of multiple base on eigen and associated vectors of a two parameter system of operators in finite-dimensional spaces is proved. Definitions of the associated vectors, multiple completeness of eigen and associated vectors of two-parameter not selfadjoint systems, nonlinearly depending on spectral parameters are introduced. At the proof of these results we essentially used the notion of the analog of an resultant of two polynomial bundles.
\end{abstract}

Keywords: Algebraic, Spectral, Resultant, Nonlinear

\section{Introduction}

The spectral theory of operators is one of essential directions of a functional analysis. Many partial equations and the equations of mathematical physics, connected with physical processes, demanded the new approach for a solution of these problems.

The method of a separation of variables for a solution of the equations with partial derivatives often appears the most comprehensible as reduces solution of the complicated equation with many variables to a solution of system of the ordinary differential equations which research is much easier.

So, for example, with help of multiparameter theory problems of a quantum mechanics $[7,15,16]$, theories of a diffraction $[9,10]$, theories of elastic envelopes, calculation of nuclear reactors [14], equilibrium processes of diffusion type [13], a Brownian motion [13], boundary value problems for the equations of elliptic-parabolic type, a Cauchy problem for the ultra parabolic equations [11], etc. are solved.

Despite of an urgency and prescription of these researches, the spectral theory of multiparameter systems is studied insufficiently. Available outcomes in this area until recently are obtained only for multiparameter selfadjoint systems of the operators linearly depending on spectral parameters.
The founder of researches of spectral problems of the multiparameter selfadjoint systems was F.V. Atkinson [1]. Studied the outcomes which are available for multiparameter symmetrical differential systems, Atkinson has constructed the spectral theory of selfadjoint multiparameter systems in finite-dimensional spaces. Further, by means of passage to the limit Atkinson has generalized the received outcomes on a case of multiparameter systems with the selfadjoint compact operators in infinite-dimensional Hilbert spaces.

In the further, it has appeared possible to build the construction of Atkinson in infinite-dimensional spaces. It has allowed construct the spectral theory of multiparameter systems in Hilbert spaces [3, 8] with help a design introduced by Atkinson for study of multiparameter systems in finite-dimensional spaces,

But, unfortunately, the technique of research in these works essentially uses only selfadjoint operators entering into multiparameter system of operators. For not selfadjoint multiparameter systems the investigated technique does not allow to solve the simplest problems of the spectral theory of operators.

The author offers the new approach to research of multiparameter problems.

In works $[5,6]$ concepts of base, multiple bases, essential to the spectral theory of multiparameter operational systems of concept of the associated vectors, completeness and multiply completeness of system of eigen and associated 
vectors are introduced.

\section{Multiparameter Spectral Theory and Its Application to Nonlinear Alge- braic System}

For the statement of existence of a solution of

$$
\begin{aligned}
& a(x, y)=a_{0}+a_{1} x+\ldots+ \\
& a_{m_{1}} x^{m_{1}}+a_{m_{1}+1} y+\ldots+\mu^{n_{1}} a_{m_{1}+n_{1}} y^{n_{1}}=0 \\
& b(x, y)=b_{0}+b_{1} x+\ldots+b_{m_{2}} x^{m_{2}} \\
& +b_{m_{2}+1} y+\ldots+b_{m_{2}+n_{2}} y^{n_{2}}=0
\end{aligned}
$$

we essentially use outcomes of author concerning the multiparameter systems, published in works [4-6] .

In the given article the multiply base property of system of eigen and associated vectors of two parameter system

$$
\begin{gathered}
\mathrm{A}(\lambda, \mu) \mathrm{x}=\left(\mathrm{A}_{0}+\lambda \mathrm{A}_{1}+\ldots+\lambda^{\mathrm{m}_{1}} \mathrm{~A}_{\mathrm{m}_{1}}\right. \\
\left.+\mu \mathrm{A}_{\mathrm{m}_{1}+1}+\ldots+\mu^{\mathrm{n}_{1}} \mathrm{~A}_{\mathrm{m}_{1}+\mathrm{n}_{1}}\right) \mathrm{x}=0 \\
\mathrm{~B}(\lambda, \mu) \mathrm{y}=\left(\mathrm{B}_{0}+\lambda \mathrm{B}_{1}+\ldots+\lambda^{\mathrm{m}_{2}} \mathrm{~B}_{\mathrm{m}_{2}}\right. \\
\left.+\mu \mathrm{B}_{\mathrm{m}_{2}+1}+\ldots+\mu^{\mathrm{n}_{2}} \mathrm{~B}_{\mathrm{m}_{2}+\mathrm{n}_{2}}\right) \mathrm{y}=0
\end{gathered}
$$

is investigated.

Below we shall reduce these outcomes as they have independent meanings. is established in tensor product $H_{1} \otimes H_{2}$ of finite-dimensional spaces $H_{1}$ and $H_{2}$

Dimension of space $H$ is the product of dimensions of spaces $H_{1}$ and $H_{2}$. In (2) linear operators $A_{i}\left(i=0,1, \ldots, m_{1}+n_{1}\right)$ act in finite-dimensional space $H_{1}$ and linear operators $B_{i}\left(i=0,1, \ldots, m_{2}+n_{2}\right)$ act in finite-dimensional space $H_{2}$.

If $f_{1} \otimes f_{2} \in H_{1} \otimes H_{2}$ and $g_{1} \otimes g_{2} \in H_{1} \otimes H_{2}$ then inner product of these elements in space $H_{1} \otimes H_{2}$ is defined by means of the formulae $\left[f_{1} \otimes f_{2}, g_{1} \otimes g_{2}\right]_{H_{1} \otimes H_{2}}=\left(f_{1}, g_{1}\right)_{H_{1}} \cdot\left(g_{1}, g_{2}\right)_{H_{2}}$

This definition is spread to other elements of tensor product spaces on linearity.

Let's reduce a series of known positions concerning the spectral theory of multiparameter system.

Definition1. $([1,3])(\lambda, \mu) \in C^{2}$ is an eigen value of the system (2) if there are nonzero elements $x \in H_{1}, y \in H_{2}$ such that (2) is fulfilled. A tensor $z=x \otimes y$ is named an eigenvector of system (2).

Definition2. An operator $A_{k}^{+}=A_{k} \otimes E_{2}$ (accordingly, $B_{k}^{+}=E_{1} \otimes B_{k}$ ) where $E_{1}$ (accordingly, $E_{2}$ ) is identical operators in $H_{1}$ (accordingly, $H_{2}$ ), names operator, induced in $H=H_{1} \otimes H_{2}$ by operators $A_{k}$ (accordingly, $B_{k}$ ).
Definition3.[5] A tensor $z_{m_{1}, m_{2}}$ name $\left(m_{1}, m_{2}\right)$ - the associated vector to an eigenvector $z_{0,0}=x \otimes y$ if following conditions are satisfied

$$
\begin{gathered}
\sum_{0 \leq r_{i} \leq k_{i}} \frac{1}{r_{1} ! r_{2} !} \frac{\partial^{r_{1}+r_{2}} A^{+}(\lambda, \mu)}{\partial^{r_{1}} \lambda \partial^{r_{2}} \mu} z_{k_{1}-r_{1}, k_{2}-r_{2}}=0 \\
\sum_{0 s_{r} \leq k,} \frac{1}{r_{1} ! r_{2} !} \frac{\partial^{r_{i}+r_{2}} A^{+}(\lambda, \mu)}{\partial^{r_{1}} \lambda \partial^{r_{2}} \mu} Z_{k_{1}-r_{1}, k_{2}-r_{2}}=0 \\
k_{s} \leq m_{s} ; i=1,2 ; s=1,2 .
\end{gathered}
$$

$\left(k_{1}, k_{2}\right)$ is arrangement from set of the whole nonnegative numbers on 2 with possible recurring and zero.

Definition4. Systems of elements $\left\{x_{k, 1}\right\}_{k}(k=1,2, \ldots, n)$ of finite-dimensional space $H_{1} \otimes H_{2}$ form $\boldsymbol{n}$-fold base in this space if any $\boldsymbol{n}$ of elements $f_{1}, \ldots, f_{n}$ of space $H_{1} \otimes H_{2}$ can be spread out in series $f_{i}=\sum_{k=1}^{s} c_{k} x_{k, i}(i=1, \ldots, n)$ with coefficients, not dependent on an index of vectors $f_{1}, \ldots, f_{n}$.

If systems $\left\{x_{k, i}\right\}_{k=1}^{r}(i=1,2, \ldots, n)$ are constructed on $\left\{x_{k, 1}\right\}_{k=1}$ with by the certain rules, equalities $f_{i}=\sum_{k=1}^{s} c_{k} x_{k, i}(i=1, \ldots, n)$ mean existence of $\boldsymbol{n}$-fold base on system of eigen and associated vectors of the system (2).

Definition5.[5]. Let be two polynomial bundles

$$
\begin{gathered}
A(\lambda)=A_{0}+\lambda A_{1}+\ldots+\lambda^{m} A_{m} \\
B(\lambda)=B_{0}+\lambda B_{1}+\ldots+\lambda^{n} B_{n},
\end{gathered}
$$

depending on the same parameter $\lambda$ and acting, generally speaking, in various Hilbert spaces $H_{1}, H_{2}$, accordingly.

The operator $\operatorname{Re} s(A(\lambda), B(\lambda))$ is under construction

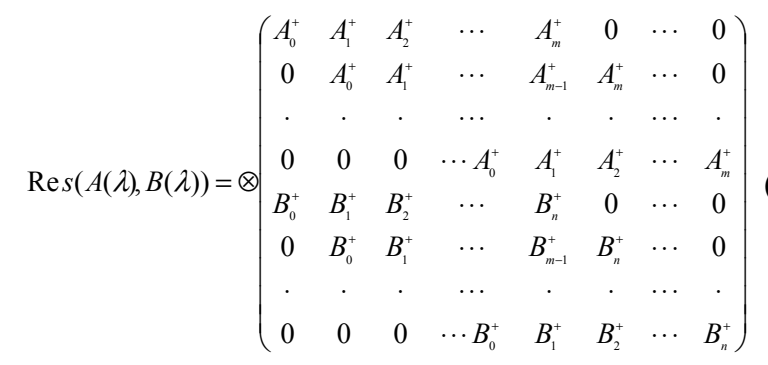

in $[2,17]$ names abstract analog of an Resultant for polynomial bundles (4).

In definition of a Resultant (5) of bundles (4) lines with operators $A_{i}$ repeated $n$ of times, and lines with operators $B_{i}$ repeated exactly $m$ of times. $m, n$ there are the highest orders of parameter $\lambda$ in bundles of $A(\lambda),(B(\lambda))$, accordingly. Thus, the Resultant is an operator, acting in space $\left(H_{1} \otimes H_{2}\right)^{m+n}$ that there is a direct sum 
$m+n$ of copies of tensor product spaces $H_{1} \otimes H_{2}$. Value of $\operatorname{Re} s(A(\lambda), B(\lambda))$ is equal to its formal expansion when each term of this expansion is tensor product of operators. Let operator $A_{m}$, or $B_{n}$, is invertible. If the highest orders of parameter $\lambda$ in bundles of $A(\lambda)$ and $B(\lambda)$ coincide (see [17]) or if the highest orders of parameter $\lambda$ in bundles of $A(\lambda)$ and $B(\lambda)$ ), generally speaking, can not coincide (see [2]) bundles (4) have a common point of spectra in the only case when the Resultant of these bundles has nonzero kernel. It is natural, that in case of when bundles act in finite-dimensional spaces, this common point of spectra of these bundles is a common eigen value of these bundles.

Theorem. Let operators $A_{i}\left(i=0,1, \ldots, m_{1}+n_{1}\right)$ also $B_{i}\left(i=0,1, \ldots, m_{2}+n_{2}\right)$ act in finite-dimensional spaces $H_{1}$ and $H_{2}$, accordingly, and one of three following conditions is fulfilled:

$$
\begin{gathered}
\text { a) } \max \left(m_{1} n_{2}, m_{2} n_{1}\right)=m_{1} n_{2}, \\
\operatorname{Ker} A_{m_{1}}=\{\theta\}, \operatorname{Ker} B_{m_{2}+n_{2}}=\{\theta\} ;
\end{gathered}
$$

$A_{m_{1}}, B_{m_{2}+n_{2}}$ are selfadjoint operators everyone in their space

$$
\begin{gathered}
\text { b) } \max \left(m_{1} n_{2}, m_{2} n_{1}\right)=m_{2} n_{1}, \\
\operatorname{Ker} B_{m_{2}}=\{\theta\}, \operatorname{Ker} A_{m_{1}+n_{1}}=\{\theta\}
\end{gathered}
$$

operators $B_{m_{2}} A_{m_{1}+n_{1}}$ are selfadjoint everyone in their spaces

c) $m_{1} n_{2}=m_{2} n_{1}$

$\operatorname{Ker}\left(A_{m_{1}}^{n_{2}} \otimes B_{m_{2}+n_{2}}^{n_{1}}+(-1)^{n_{1} n_{2}} A_{m_{1}+n_{1}}^{n_{2}} \otimes B_{m_{2}}^{n_{1}}\right)=\{\theta\}$,

$A_{m_{1}}, B_{m_{2}}, A_{m_{1}+n_{1}}, B_{m_{2}+n_{2}}$ are the selfadjoint operators, acting everyone in its finite-dimensional space.

Then the $\max \left(m_{1} n_{2}, m_{2} n_{1}\right)$-fold base on system of eigen and associated vectors of (4) takes place.

Proof. It is fixed one of parameters in (4). For a determinacy, we shall suppose, that it is parameter $\lambda$ and $\lambda=\lambda_{0}$. Then it is received two bundles, each of which depends on one parameter $\mu$.

$$
\begin{gathered}
\mathrm{A}\left(\lambda_{0}, \mu\right)=\mathrm{A}_{0}+\lambda_{0} \mathrm{~A}_{1}+\ldots+ \\
\lambda_{0}{ }^{\mathrm{m}_{1}} \mathrm{~A}_{\mathrm{m}_{1}}+\mu \mathrm{A}_{\mathrm{m}_{1}+1}+\ldots+\mu^{\mathrm{n}_{1}} \mathrm{~A}_{\mathrm{m}_{1}+\mathrm{n}_{1}} \\
\mathrm{~B}\left(\lambda_{0}, \mu\right)=\mathrm{B}_{0}+\lambda_{0} \mathrm{~B}_{1}+\ldots+ \\
\lambda_{0}{ }^{\mathrm{m}_{2}} \mathrm{~B}_{\mathrm{m}_{2}}+\mu \mathrm{B}_{\mathrm{m}_{2}+1}+\ldots+\mu^{\mathrm{n}_{2}} \mathrm{~B}_{\mathrm{m}_{2}+\mathrm{n}_{2}}
\end{gathered}
$$

We introduce labels

$$
\widetilde{A}\left(\lambda_{0}\right)=A_{0}+\lambda_{0} A_{1}+\ldots+\lambda_{0}^{m_{1}} A_{m_{1}},
$$

$$
\widetilde{B}\left(\lambda_{0}\right)=B_{0}+\lambda_{0} B_{1}+\ldots+\lambda_{0}^{m_{2}} B_{m_{2}}
$$

Then an Resultant of bundles

$$
\begin{aligned}
A\left(\lambda_{0}, \mu\right) & =\widetilde{A}\left(\lambda_{0}\right)+\mu A_{m_{1}+1}+\ldots+\mu^{n_{1}} A_{m_{1}+n_{1}} \\
B\left(\lambda_{0}, \mu\right) & =\widetilde{B}\left(\lambda_{0}\right)+\mu B_{m_{2}+1}+\ldots+\mu^{n_{2}} B_{m_{2}+n_{2}},
\end{aligned}
$$

depending on one parameter $\mu$ looks like:

$$
\begin{aligned}
& \operatorname{Re} s\left(A\left(\lambda_{0}, \mu\right), B\left(\lambda_{0}, \mu\right)\right)=
\end{aligned}
$$

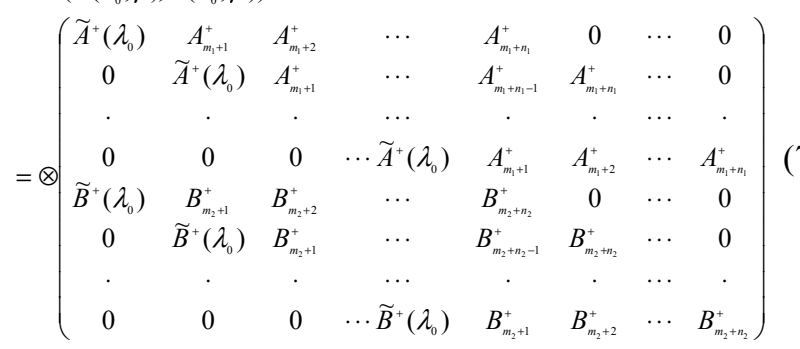

In (7) lines with operators $A_{i}$ are repeated $n_{2}$ of times, and lines with operators $B_{i}$ are repeated $n_{1}$ of times. Thus, the Resultant $\operatorname{Re} s\left(A\left(\lambda_{0}, \mu\right), B\left(\lambda_{0}, \mu\right)\right)$ is an operator in space $\left(H_{1} \otimes H_{2}\right)^{n_{1}+n_{2}}$ that there is a direct sum $n_{1}+n_{2}$ of copies of tensor product spaces $H_{1} \otimes H_{2}$.

From outcomes of work [2] follow, that spectra of operators

$$
\begin{gathered}
A\left(\lambda_{0}, \mu\right)=A_{0}+\lambda_{0} A_{1}+\ldots+\lambda_{0}^{m_{1}} A_{m_{1}}+\mu A_{m_{1}+1}+\ldots+\mu^{n_{1}} A_{m_{1}+m_{1}}, \\
B\left(\lambda_{0}, \mu\right)=B_{0}+\lambda_{0} B_{1}+\ldots+\lambda_{0}^{m_{2}} B_{m_{2}}+\mu B_{m_{2}+1}+\ldots+\mu^{m_{2}} B_{m_{2}+n_{2}}
\end{gathered}
$$

intersect if and only if $\operatorname{Ker} R\left(A\left(\lambda_{0}\right), B\left(\lambda_{o}\right)\right) \neq\{\theta\}$.

As operators $A(\lambda, \mu), B(\lambda, \mu)$ act in finite-dimensional spaces this common point of their spectra can be only common eigen value of these bundles. The last means, that the two parameter system (2) has an eigen value $\left(\lambda_{0}, \mu_{0}\left(\lambda_{0}\right)\right)$. If the element $z=\left(\widetilde{x}_{1}, \widetilde{x}_{2}, \ldots, \widetilde{x}_{n_{1}+n_{2}}\right) \in\left(H_{1} \otimes H_{2}\right)^{n_{1}+n_{2}}$ belongs to the kernel of an Resultant $\operatorname{Re} s\left(A\left(\lambda_{0}, \mu\right), B\left(\lambda_{0}, \mu\right)\right)$, then

$$
\begin{gathered}
\widetilde{A}^{+}\left(\lambda_{0}\right) \widetilde{x}_{1}+\widetilde{A}_{m_{1}+1}^{+} \widetilde{x}_{2}+\ldots+\widetilde{A}_{m_{1}+n_{1}}^{+} \widetilde{x}_{n_{1}}=0 \\
\widetilde{A}^{+}\left(\lambda_{0}\right) \widetilde{x}_{2}+\widetilde{A}_{m_{1}+1}^{+} \widetilde{x}_{3}+\ldots+\widetilde{A}_{m_{1}+n_{1}}^{+} \widetilde{x}_{n_{1}+1}=0 \\
\widetilde{A}^{+}\left(\lambda_{0}\right) \widetilde{x}_{n_{2}}+\widetilde{A}_{m_{1}+1}^{+} \widetilde{x}_{n_{2}+1}+\ldots+\widetilde{A}_{m_{1}+n_{1}}^{+} \widetilde{x}_{n_{1}+n_{2}}=0 \\
\widetilde{B}^{+}\left(\lambda_{0}\right) \widetilde{x}_{1}+\widetilde{B}_{m_{2}+1}^{+} \widetilde{x}_{2}+\ldots+\widetilde{B}_{m_{2}+n_{2}}^{+} \widetilde{x}_{n_{2}}=0 \\
\widetilde{B}^{+}\left(\lambda_{0}\right) \widetilde{x}_{2}+\widetilde{B}_{m_{2}+1}^{+} \widetilde{x}_{3}+\ldots+\widetilde{B}_{m_{2}+n_{2}}^{+} \widetilde{x}_{n_{2}+1}=0 \\
\widetilde{B}^{+}\left(\lambda_{0}\right) \widetilde{x}_{n_{1}}+\widetilde{B}_{m_{2}+1}^{+} \widetilde{x}_{m_{1}+1}+\ldots+\widetilde{B}_{m_{2}+n_{2}}^{+} \widetilde{x}_{n_{1}+n_{2}}=0
\end{gathered}
$$

By sequential elimination of elements $\widetilde{x}_{n_{n+2-1}} \in H_{1} \otimes H_{2}$, 
$\widetilde{x}_{2} \in H_{1} \otimes H_{2}$ from system of equalities (8), we come to some equation in tensor product space $H_{1} \otimes H_{2}$. To the similar equation we come by representation of value of Resultant $\operatorname{Re} s\left(A\left(\lambda_{0}\right), B\left(\lambda_{0},\right)\right.$.

By virtue of bulkiness of the received equation operational coefficients at degrees $\lambda^{i}$ we shall designate through $\widetilde{C}_{i}\left(i=1,2, \ldots, \max \left(m_{1} n_{2}, m_{2} n_{1}\right)\right)$.

Let's introduce a label $\max \left(m_{1} n_{2}, m_{2} n_{1}\right)=r$.Thus, we come to some equation

$\widetilde{C}\left(\lambda_{0}\right) \widetilde{x}_{1}=\widetilde{C}_{0} \widetilde{x}_{1}+\lambda_{0} \widetilde{C}_{1} \widetilde{x}_{1}+\ldots+\lambda_{0}{ }^{r} \widetilde{C}_{r} \widetilde{x}_{1}=0$, acting in a tensor product space $H=H_{1} \otimes H_{2}$. As the parameter $\lambda$ is fixed arbitrarily, we consider a bundle

$$
\widetilde{C}(\lambda) \widetilde{x}_{1}=\widetilde{C}_{0} \widetilde{x}_{1}+\lambda \widetilde{C}_{1} \widetilde{x}_{1}+\ldots+\lambda^{r} \widetilde{C}_{r} \widetilde{x}_{1}=0
$$

in finite-dimensional space $H=H_{1} \otimes H_{2}$.

From outcomes of the spectral theory of operators follows, that the received bundle (9) in finite-dimensional space has $r$-fold base on system of its eigen and associated vectors. Really, at realization of conditions Theorem are satisfied all conditions of the Theorem of Keldysh [12], concerning multiply completeness of system of eigen and associated vectors of polynomial bundle

$\widetilde{C}(\lambda)=\widetilde{C}_{0}+\widetilde{C}_{1}+. .+\lambda \widetilde{C}_{r}$

The theorem of Keldysh is following: let $T_{n}$ is selfadjoint completely continuous operator of the finite order $\left(T_{n}\right.$ $\left.\in \sigma_{p}\right), \operatorname{Ker} T_{n}=\{\theta\}$, operators $T_{i} T_{n}^{-\frac{i}{n}}(i=0,1, \ldots, n-1)$ completely continuous operators then $n$-fold completeness of system of eigen and associated vectors of the operational bundle $T(\lambda)=T_{0}+\lambda T_{1}+\ldots+\lambda^{n} T_{n}$, acting in a Hilbert space, takes place.

Let's remind, that under an eigen value of a polynomial bundle $T(\lambda)$ such the complex number $\lambda_{0}$ is understood, that for it exists such nonzero vector $x_{0}$, that $T\left(\lambda_{0}\right) x_{0}=x_{0}$, $m$-th associated vector $x_{k}$ to an eigenvector $x_{0}$ is the vector, satisfying to conditions

$$
\begin{aligned}
& \mathrm{x}_{\mathrm{k}}=\mathrm{T}\left(\lambda_{0}\right) \mathrm{x}_{\mathrm{k}}+\frac{\mathrm{d}}{\mathrm{d} \lambda} \mathrm{T}\left(\lambda_{0}\right) \mathrm{x}_{\mathrm{k}-1} \\
& +\ldots+\frac{1}{\mathrm{k} !} \frac{\mathrm{d}^{\mathrm{k}}}{\mathrm{d} \lambda^{\mathrm{k}}} \mathrm{T}\left(\lambda_{0}\right) \mathrm{x}_{0} ; \mathrm{k}=1, \ldots, \mathrm{m}
\end{aligned}
$$

Let $\left\{x_{k, 0}\right\}$ be a system of eigen and associated vectors of a bundle $T(\lambda)$, then derivative systems of vectors on system of eigen and associated vectors of polynomial bundle $T(\lambda)$ are constructed by rules

$$
\mathrm{x}_{\mathrm{i}, \mathrm{j}}=\left\{\begin{array}{l}
\frac{\mathrm{d}^{\mathrm{j}}}{\mathrm{dt}^{\mathrm{j}}} \mathrm{e}^{\lambda_{0} \mathrm{t}}\left(\mathrm{x}_{\mathrm{i}, 0}+\right. \\
\mathrm{x}_{\mathrm{i}-1,0} \frac{\mathrm{t}}{1 !}+\ldots+\mathrm{x}_{0,0} \frac{\mathrm{t}^{\mathrm{i}}}{\mathrm{i} !}
\end{array}\right\}_{\mathrm{t}=0} i=1,2, \ldots
$$

In case of finite-dimensional space all conditions of the Theorem of Keldysh

are fulfilled ,consequently, system of eigen and associated vectors of a bundle $\widetilde{C}(\lambda)=\widetilde{C}_{0}+\lambda \widetilde{C}_{1}+\ldots+\lambda \widetilde{C}_{r}$ forms $r$-fold base in space $H_{1} \otimes H_{2}$.

Really, at realization of a conditions a) of the Theorem coefficient at $\lambda^{r}$ is an operator $\widetilde{C}_{r}=A_{m_{1}}^{n_{2}} \otimes B_{m_{2}+n_{2}}^{n_{1}}$. It is easy to see, that it is selfadjoint operator and $\operatorname{Ke}\left(A_{m_{1}}^{m_{1}} \otimes B_{m_{2}+n_{2}}\right)=\{\theta$;

At realization of conditions $\mathrm{b}$ ) of the Theorem it is had, that $\operatorname{Ker}_{m_{1} n_{1}}^{n_{1}} \otimes B_{m_{2}}^{n}=\left\{\theta_{\}}\right.$, operator $A_{m_{1}+n_{1}}^{n_{2}} \otimes B_{m_{2}}^{n_{1}}$ is selfadjoint, and, at last, at realization of conditions c) of the Theorem the operator $A_{m_{1}+n_{1}}^{n_{2}} \otimes B_{m_{2}}^{n_{1}}+(-1)^{n_{1} n_{2}} A_{m_{1}+n_{1}}^{n_{2}} \otimes B_{m_{2}}^{n_{1}}$ is selfadjoint and $\operatorname{Ker}\left(A_{m_{1}+n_{1}}^{n_{2}} \otimes B_{m_{2}}^{n_{1}}+(-1)^{n_{1} n_{2}} A_{m_{1}}^{n_{2}} \otimes B_{m_{2}+n_{2}}^{n_{1}}\right)=\{\theta\}$ also. In all these three cases at coefficient at $\lambda^{r}$ of bundle $\widetilde{C}\left(\lambda_{0}\right)$ is selfadjoint invertible operator.

Remains to prove concurrence of system of eigen and associated vectors of a two-parameter problem (2) and systems of eigen and associated vectors of the polynomial bundle (9) received by the above-stated way in tensor product space $H_{1} \otimes H_{2}$.

Let $\widetilde{x}$ be an eigenvector of the equation (9). Supposing $\tilde{x}_{1}=\tilde{x}$ and using that fact, that (9) there is an expansion of a Resultant (4), we determine vectors $\widetilde{x}_{2}, \widetilde{x}_{3}, \ldots, \widetilde{x}_{n_{1}+n_{2}}$. Besides from (8) it is had, that an element $\left(\tilde{x}_{1}, \tilde{x}_{2}, \widetilde{x}_{3}, \ldots\right.$, $\left.\tilde{x}_{n_{1}+n_{2}}\right) \in\left(H_{1} \otimes H_{2}\right)^{n_{1}+n_{2}}$ enters into the kernel of a Resultant $\operatorname{Re} s\left(A\left(\lambda_{0}, \mu\right), B\left(\lambda_{0}, \mu\right)\right)$ of bundles $A\left(\lambda_{0}, \mu\right)$ and $B\left(\lambda_{0}, \mu\right)$.

In works $[5,6]$ the subsequent components of the ele$\operatorname{ment}\left(\widetilde{x}_{1}, \widetilde{x}_{2}, \widetilde{x}_{3}, \ldots, \widetilde{x}_{n_{1}+n_{2}}\right)$ entering into the kernel of Resultant $\operatorname{Re} s\left(A\left(\lambda_{0}\right), B\left(\lambda_{0}\right)\right)$ are for the first time well enough studied . In particularities, in case of when operational bundles act in finite-dimensional space and depend on the same parameters, the aspect of the first component $\widetilde{x}_{1}$ of the element $\left(\widetilde{x}_{1}, \widetilde{x}_{2}, \widetilde{x}_{3}, \ldots, \widetilde{x}_{n_{1}+r_{2}}\right) \in\left(H \otimes H_{2}\right)^{n_{1+n_{2}}}$, entering into the kernel of a Resultant $\operatorname{Res}\left(A\left(\lambda_{0}, \mu\right), B\left(\lambda_{0}, \mu\right)\right)$ is constructed by the following manner : $\widetilde{x}_{1}$ has the form linear combinations of elements $U_{i} \otimes V_{0}+U_{i-1} \otimes V_{1}+\ldots+U_{0} \otimes V_{i}$, where $U_{0}, \ldots, U_{i}$ (accordingly, $\left.V_{0}, \ldots, V_{i}\right)$ is a restricted chain of eigen and associated vectors of the bundle $A\left(\lambda_{0}, \mu\right)$ (accordingly, $B\left(\lambda_{0}, \mu\right)$ ) at the fixed value of parameter $\lambda=\lambda_{0}$. The parameter $\mu$, being some common eigen value $\mu\left(\lambda_{0}\right)$ both of operators $A\left(\lambda_{0}, \mu\right)$ and $B\left(\lambda_{0}, \mu\right) . U_{0}, \ldots, U_{m}$ (accordingly, $\left.V_{0}, \ldots, V_{s}\right)$ system of eigen and associated vectors of an operator $A\left(\lambda_{0}, \mu\right)$ (accordingly, $B\left(\lambda_{0}, \mu\right)$ ), ad equating to their common eigen value $\mu\left(\lambda_{0}\right)$.

The second and following components of the element 
$\left(\widetilde{x}_{1}, \widetilde{x}_{2}, \widetilde{x}_{3}, \ldots, \tilde{x}_{n_{1}+n_{2}}\right)$ coincide with the elements of derivative systems constructed on the first components $U_{i} \otimes V_{0}+U_{i-1} \otimes V_{1}+\ldots+U_{0} \otimes V_{i}$. Eigen values $\left(\lambda_{0}, \mu\left(\lambda_{0}\right)\right)$ of twoparameter system (2) can be several and all they have in the first coordinate number $\lambda_{0}$.

The form of the first component of element entering the kernel of the resultant of a two polynomial bundles from one parameter acting in finite-dimensional spaces is announced in [2]. For two-parameter systems in Hilbert spaces, at realization of some conditions the aspect of the first coordinate $\widetilde{x}_{1}$ of an element $\left(\widetilde{x}_{1}, \widetilde{x}_{2}, \widetilde{x}_{3}, \ldots\right.$, $\left.\widetilde{x}_{n_{1}+n_{2}}\right) \in\left(H_{1} \otimes H_{2}\right)^{n_{1}+\eta_{2}}$, entering into the kernel of a Resultant $\operatorname{Res}\left(A\left(\lambda_{0}, \mu\right), B\left(\lambda_{0}, \mu\right)\right)$ is announced in [5].

We shall show, that each eigenvector of the equation $\widetilde{C}\left(\lambda_{0}\right) \tilde{x}=0$ is the first component of an element, entering into the kernel of Resultant of bundles $A\left(\lambda_{0}, \mu\right)$ and $B\left(\lambda_{0}, \mu\right)$. Really, considering $\widetilde{x}=\widetilde{x}_{1}$, we have that the left part of the equation $\widetilde{C}\left(\lambda_{0}\right) \widetilde{x}=0$ represents expansion of a Resul$\operatorname{tant}(7)$ on a vector $\widetilde{x}$.

Converting to definition of eigen and associated vectors of two-parameter system, from (9) we discover, that the element $\tilde{x}=\tilde{x}_{1}$ can be an eigenvector of two-parameter system of operators (3), ad equating to an eigen value $\left(\lambda_{0}, \mu\left(\lambda_{0}\right)\right)$ or the associated vector of this system (3) ad equating to some eigen value $\left(\lambda_{0}, \mu\left(\lambda_{0}\right)\right)$ in which definition there is no derivation on the parameter $\lambda$.

Such associated vectors of two-parameter system would be expedient for naming the associated vectors of two-parameter system in a direction $\boldsymbol{\mu}$. Similarly, associated vectors in which definition there is no derivation on parameter $\boldsymbol{\mu}$, we shall name the associated vectors of two-parameter system in a direction $\lambda$.

Thus, all eigenvectors of the equation (9) corresponding to eigen value $\lambda_{0}$ are either eigen vector or associated vector on direction $\boldsymbol{\mu}$ of the twoparameter system corresponding to an eigen value $\left(\lambda_{0}, \mu\left(\lambda_{0}\right)\right)$.

Remains to prove, that all associated vectors of (9) also be the associated vectors of a two-parameter problem (2).

Let $\widetilde{y}_{1}$ there is a first associated vector to an eigenvector $\widetilde{x}_{1}$ of the equation (9)

corresponding to its eigen value $\lambda_{0}$. Then we have

$$
\begin{aligned}
& \tilde{\mathrm{C}}\left(\lambda_{0}\right) \tilde{\mathrm{y}}_{1}+\frac{\mathrm{d}}{\mathrm{d} \lambda} \tilde{\mathrm{C}}\left(\lambda_{0}\right) \tilde{\mathrm{x}}_{1} \\
& =\left(\tilde{\mathrm{C}}_{0}+\lambda_{0} \tilde{\mathrm{C}}_{1}+\ldots+\lambda_{0}{ }^{\mathrm{r}} \tilde{\mathrm{C}}_{\mathrm{r}}\right) \tilde{\mathrm{y}}+ \\
& +\tilde{\mathrm{C}}_{1} \tilde{\mathrm{x}}_{1}+\ldots+\mathrm{r} \lambda_{0}^{\mathrm{r}-1} \tilde{\mathrm{C}}_{\mathrm{r}} \tilde{\mathrm{x}}_{1},\left(\max \left(\mathrm{m}_{1} \mathrm{n}_{2}, \mathrm{~m}_{2} \mathrm{n}_{1}\right)=\mathrm{r}\right)
\end{aligned}
$$

$$
\widetilde{C}\left(\lambda_{0}\right) \widetilde{x}_{1}=\widetilde{C} \widetilde{x}_{1}+\lambda_{0} \widetilde{C}_{1} \widetilde{x}_{1}+\ldots+\lambda_{0}^{r} \widetilde{C_{r}} \widetilde{x}_{1}=0
$$

Equation (11) means that there is nonzero element $\left(\widetilde{x}_{1}, \widetilde{x}_{2}, \ldots, \widetilde{x}_{n_{1}+n_{2}}\right) \in H^{n_{1}+n_{2}}$, entering into the kernel of Resultant of bundles

$$
\begin{aligned}
& A\left(\lambda_{0}, \mu\right)=A_{0}+\lambda_{0} A_{1}+\ldots+\lambda_{0}^{m_{1}} A_{m_{1}}+\mu A_{m_{1}+1}+\ldots+\mu^{n_{1}} A_{m_{1}+n_{1}}, \\
& B\left(\lambda_{0}, \mu\right)=B_{0}+\lambda_{0} B_{1}+\ldots+\lambda_{0}^{m_{2}} B_{m_{2}}+\mu B_{m_{2}+1}+\ldots+\mu^{n_{2}} B_{m_{2}+n_{2}}
\end{aligned}
$$

such that (8) is fulfilled.

It is clear that all eigen vectors of (6) coincide with the 1 -st component of element from the $\operatorname{Ker} \operatorname{Re} s\left(A\left(\lambda_{0}, \mu\right), B\left(\lambda_{0}, \mu\right)\right)$. We have the completeness of eigen and associated vectors of the $\operatorname{Re} s\left(A\left(\lambda_{0}, \mu\right), B\left(\lambda_{0}, \mu\right)\right)$ in $\operatorname{space}\left(H_{1} \otimes H_{2}\right)^{n_{1}+n_{2}}$

$$
\text { If }\left(\tilde{y}=\left(\tilde{y}_{1}, \tilde{y}_{2}, \ldots, \tilde{y}_{n_{1}+n_{2}}\right) \in H^{n_{1}+n_{2}}\right. \text { the 1-st associated }
$$
vector to eigen vector $\left(\tilde{x}_{1}, \tilde{x}_{2}, \ldots, \tilde{x}_{n_{1}+n_{2}}\right) \in H^{n_{1}+n_{2}}$ of the operator $\operatorname{Re} s\left(A\left(\lambda_{0}, \mu\right), B\left(\lambda_{0}, \mu\right)\right)$ then we have that the following expressions (12) and equalities (8) are fulfilled

$$
\begin{aligned}
& \tilde{\mathrm{A}}^{+}\left(\lambda_{0}\right) \tilde{\mathrm{y}}_{1}+\tilde{\mathrm{A}}_{\mathrm{m}_{1}+1}^{+} \tilde{\mathrm{y}}_{2}+\ldots+ \\
& \tilde{\mathrm{A}}_{\mathrm{m}_{1}+\mathrm{n}_{1}}^{+} \tilde{\mathrm{y}}_{\mathrm{n}_{1}}+\frac{\mathrm{d}}{\mathrm{d} \lambda} \tilde{\mathrm{A}}\left(\lambda_{0}\right) \tilde{\mathrm{x}}_{1}=0 \\
& \tilde{\mathrm{A}}^{+}\left(\lambda_{0}\right) \tilde{\mathrm{y}}_{2}+\tilde{\mathrm{A}}_{\mathrm{m}_{1}+1}^{+} \tilde{\mathrm{y}}_{3}+\ldots+ \\
& \tilde{\mathrm{A}}_{\mathrm{m}_{1}+\mathrm{n}_{1}}^{+} \tilde{\mathrm{y}}_{\mathrm{n}_{1}+1}+\frac{\mathrm{d}}{\mathrm{d} \lambda} \tilde{\mathrm{A}}\left(\lambda_{0}\right) \tilde{\mathrm{x}}_{2}=0 \\
& \tilde{\mathrm{A}}^{+}\left(\lambda_{0}\right) \tilde{\mathrm{y}}_{\mathrm{n}_{2}}+\tilde{\mathrm{A}}_{\mathrm{m}_{1}+1}^{+} \tilde{\mathrm{y}}_{\mathrm{n}_{2}+1}+\ldots+ \\
& \tilde{\mathrm{A}}_{\mathrm{m}_{1}+\mathrm{n}_{1}}^{+} \tilde{\mathrm{y}}_{\mathrm{n}_{1}+\mathrm{n}_{2}}+\frac{\mathrm{d}}{\mathrm{d} \lambda} \tilde{\mathrm{A}}\left(\lambda_{0}\right) \tilde{\mathrm{x}}_{\mathrm{n}_{2}}=0 \\
& \tilde{\mathrm{B}}^{+}\left(\lambda_{0}\right) \tilde{\mathrm{y}}_{1}+\tilde{\mathrm{B}}_{\mathrm{m}_{2}+1}^{+} \tilde{\mathrm{y}}_{2}+\ldots+ \\
& \tilde{\mathrm{B}}_{\mathrm{m}_{2}+\mathrm{n}_{2}}^{+} \tilde{\mathrm{y}}_{\mathrm{n}_{2}}+\frac{\mathrm{d}}{\mathrm{d} \lambda} \tilde{\mathrm{B}}\left(\lambda_{0}\right) \tilde{\mathrm{x}}_{1}=0 \\
& \tilde{\mathrm{B}}^{+}\left(\lambda_{0}\right)^{+} \tilde{\mathrm{y}}_{\mathrm{n}_{1}}+\tilde{\mathrm{B}}_{\mathrm{m}_{2}+1}^{+} \tilde{\mathrm{y}}_{\mathrm{n}_{1}+1}+\ldots+ \\
& \tilde{\mathrm{B}}_{\mathrm{m}_{2}+\mathrm{n}_{2}}^{+} \tilde{\mathrm{y}}_{\mathrm{n}_{1}+\mathrm{n}_{2}}+\frac{\mathrm{d}}{\mathrm{d} \lambda} \tilde{\mathrm{B}}\left(\lambda_{0}\right) \tilde{\mathrm{x}}_{\mathrm{n}_{1}}=0 \\
& \tilde{\mathrm{A}}^{+}\left(\lambda_{0}\right) \tilde{\mathrm{X}}_{1}+\tilde{\mathrm{A}}_{\mathrm{m}_{1}+1}^{+} \tilde{\mathrm{x}}_{2}+\ldots+\tilde{\mathrm{A}}_{\mathrm{m}_{1}+\mathrm{n}_{1}}^{+} \tilde{\mathrm{x}}_{\mathrm{n}_{1}}=0 \\
& \widetilde{A}^{+}\left(\lambda_{0}\right) \widetilde{x}_{n_{2}+1}+\widetilde{A}_{m_{1}+1}^{+} \widetilde{x}_{n_{2}+1}+\ldots+\widetilde{A}_{m_{1}+n_{1}}^{+} \widetilde{x}_{n_{1}+n_{2}}=0
\end{aligned}
$$

and 


$$
\begin{aligned}
& \mathrm{B}^{+}\left(\lambda_{0}\right) \tilde{\mathrm{x}}_{1}+\tilde{\mathrm{B}}_{\mathrm{m}_{1}+1}^{+} \tilde{\mathrm{x}}_{2}+\ldots+\tilde{\mathrm{B}}_{\mathrm{m}_{2}+\mathrm{n}_{2}}^{+} \tilde{\mathrm{x}}_{\mathrm{n}_{2}}=0 \\
& B^{+}\left(\lambda_{0}\right) \widetilde{x}_{n_{1}}+\widetilde{B}_{n_{2}+2}^{+} \widetilde{x}_{n_{1}+1}+\ldots+\widetilde{B}_{m_{2}+n_{2}}^{+} \widetilde{x}_{n_{1}+n_{2}}=0
\end{aligned}
$$

The last means, that $\widetilde{y}_{1}$ there is the associated vector of two-parameter system corresponding to eigenvector $\widetilde{x}_{1}$ with eigen value $\left(\lambda_{0}, \mu\left(\lambda_{0}\right)\right)$. From (8) we obtain (11). From (12) and (13) we obtain (10).By analogy in the image it is possible to prove, that the associated vectors of other orders of the equations (9) corresponding to an eigen value $\lambda_{0}$ also are the associated vectors of two-parameter system (2). At each eigen value $\lambda_{0}$ of the equation (9) corresponding to $\lambda_{0}$ eigen and associate vectors of (9) coincide with eigen and associated vectors of the two-parameter system (2), corresponding some eigen values $\left(\lambda_{0}, \mu\left(\lambda_{0}\right)\right)$ of system (2) . Eigen vector of the equation (9) is eigenvector of (2) or its associated vector on direction $\mu$. Associated vectors of the equation (9),corresponding to eigen value $\lambda_{0}$ are also associated vectors of the system (2). Earlier we proved that the system of all eigen and associated vectors of the equation (9) forms the $\max \left(m_{1} n_{2}, m_{2} n_{1}\right)$-fold base of space $H_{1} \otimes H_{2}$. Then the system of eigen and associated vectors of two-parameter system (2) also forms $\max \left(m_{1} n_{2}, m_{2} n_{1}\right)$ -fold base in space $H_{1} \otimes H_{2}$. The Theorem is proved.

In the special case, when $H_{1}=H_{2}=R$ and operators $A_{i}, B_{k},\left(i=1,2, \ldots, m_{1}+n_{1} ; k=1,2, \ldots, m_{2}+n_{2}\right)$ are real numbers.

If to consider variables $x$ and $y$ parameters then the algebraic system (1) is a special case of multiparameter system (2).

Corollary. Let one of three following conditions is fulfilled:
a) $\max \left(m_{1} n_{2}, m_{2} n_{1}\right)=m_{1} n_{2}, a_{m_{1}} \neq 0, b_{m_{2}+n_{2}} \neq 0$;
b) $\max \left(m_{1} n_{2}, m_{2} n_{1}\right)=m_{2} n_{1}, b_{m_{2}} \neq 0, a_{m_{1}+n_{1}} \neq 0$
c) $m_{1} n_{2}=m_{2} n_{1}$ is $a_{m_{1}+n_{1}}^{n_{2}} b_{m_{2}}^{n_{1}}+(-1)^{n_{1} n_{2}} a_{m_{1}}^{n_{2}} b_{m_{2}+n_{2}}^{n_{1}} \neq 0$,

Then the algebraic system (1) has the solutions.

\section{Conclusion}

In this paper it is proved the existence of solutions of some nonlinear algebraic system .For the proof of this it is essentially used the results of author, obtained for the multiparameter system of operator in the finite dimensional spaces

\section{References}

[1] Atkinson F.V. Multiparameter spectral theory. Bull.Amer.Math.Soc.1968, 74, 1-27

[2] Balinskii A.I Generation of notions of Bezutiant and Resultant DAN of Ukr SSR, ser.ph.-math and tech. of sciences, 1980, 2, pp.3-6( in Russian).

[3] Browne P.J. Multiparameter spectral theory. Indiana Univ. Math. J,24, 3, 1974.

[4] Dzhabarzadeh R.M. On existence of common eigen value of some operator-bundles, that depends polynomial on parameter. International conference of topology, 3-9 oct., 1987, Baku. Tez., part 2,page 99

[5] Dzhabarzadeh R.M. Spectral theory of two parameter system in finite dimensional space. Transactions of Azerbaijan National Academy of Sciences, v.XVIII,3-4,pp.12-18

[6] Dzhabarzadeh R.M. Multiparameter spectral theory. Lambert Academic Publishing, 2 mart 2012, pp.182

[7] Prugoveĉku E. Quantum mechanics in Hilbert space. Academic Press, New York, London, 1971

[8] Sleeman B.D. Multiparameter spectral theory in Hilbert space. Pitnam Press, London, 1978, pp.118.

[9] Hargrave B.A., Sleeman B.D. The numerical solution of two-parameter eigenvalue to the problem of diffraction by a plane angular sector. J. Inst.Math. Applic.14, 1974, 9-22.

[10] Voytovich N.N., Katsenelenbaum B.Z., Sivov A.N.Generaliz a method of characteristic oscillations in the theory of a diffraction - M.: publishing "Science", 1977. - pp. 416.

[11] Genchev T.Q. (Генчев) About the ultraparabolic equations. DAS of USSR, 1963, т.151, №2, p.265-268 (in Russian)

[12] Keldysh M.V. About completeness of eigenfunctions of some classes of self-conjugate linear operators. UMN, 1971, т.27, issue 4, p.15-41.(in Russian)

[13] Levi P. (Леви). Stochastic processes and a Brownian motion. M.: publishing "Science", 1979, pp 375 (in Russian).

[14] MarchukG.I. (Марчук) . Metody of calculation of nuclear reactors. M.: , "State Atom Publishing",1961 (in Russian).

[15] Richmakher R (Рихмахер). Printsipy of modern mathematical physics., M. publishing "Mir", 1982,p. 486 (in Russian).

[16] Fok V.A. (Фок). The beqinnings of a quantum mechanics. M.: Publishing "Science", 1976, pp,376.

[17] Khayniq Q (Хайниг). Abstract analog of an eliminant of two polynomial bundles. The Functional analysis and its applications, 1977, 2, issue 3, pp.94-95. (in Russsian) 